\title{
The biological context of HIV-1 host interactions reveals subtle insights into a system hijack
}

\author{
Jonathan E Dickerson'1, John W Pinney² and David L Robertson*1
}

\begin{abstract}
Background: In order to replicate, HIV, like all viruses, needs to invade a host cell and hijack it for its own use, a process that involves multiple protein interactions between virus and host. The HIV-1, Human Protein Interaction Database available at NCBI's website captures this information from the primary literature, containing over 2,500 unique interactions. We investigate the general properties and biological context of these interactions and, thus, explore the molecular specificity of the HIV-host perturbation. In particular, we investigate (i) whether HIV preferentially interacts with highly connected and 'central' proteins, (ii) known phenotypic properties of host proteins inferred from essentiality and disease-association data, and (iii) biological context (molecular function, processes and location) of the host proteins to identify attributes most strongly associated with specific HIV interactions.

Results: After correcting for ascertainment bias in the literature, we demonstrate a significantly greater propensity for HIV to interact with highly connected and central host proteins. Unexpectedly, we find there are no associations between HIV interaction and inferred essentiality. Similarly, we find a tendency for HIV not to interact with proteins encoded by genes associated with disease. Crucially, we find that functional categories over-represented in HIV-host interactions are innately enriched for highly connected and central proteins in the host system.

Conclusions: Our results imply that HIV's propensity to interact with highly connected and central proteins is a consequence of interactions with particular cellular functions, rather than being a direct effect of network topological properties. The lack of a propensity for interactions with phenotypically essential proteins suggests a selective pressure to minimise virulence in retroviral evolution. Thus, the specificity of HIV-host interactions is complex, and only superficially explained by network properties.
\end{abstract}

\section{Background}

Human immunodeficiency virus type 1 (HIV-1) and its associated illnesses have major health and socio-economic impacts, particularly in developing countries [1]. Concomitant with the progression of the HIV pandemic there has, thus, been a major international research effort, leading to a detailed understanding of HIV biology. One of the most important aspects of this knowledge is the set of known contacts between viral proteins and the host system[2-4], fundamental to HIV's life cycle. HIV, like all viruses, subjugates and exploits host cells in order to propagate. To achieve this, the HIV virion must first bind to a host cell, primarily CD4+ T cells, macrophages and dendritic cells, and then 'hijack' their cellular

* Correspondence: david.robertson@manchester.ac.uk

1 Faculty of Life Sciences, University of Manchester, Oxford Road, Manchester, M13 9PT, UK

Full list of author information is available at the end of the article machinery [5]. Untreated HIV infection leads to a decrease in CD4+ T cell count, eventually resulting in the loss of cell-mediated immunity, an immunocompromised state and the onset of AIDS (Acquired Immunodeficiency Syndrome) [6]. However, infection with the HIV-like simian immunodeficiency virus (SIV) in its "natural" hosts, does not generally result in the development of AIDS, even when viral loads are high [7]. Despite SIV exhibiting high viral loads, and there being a decreased CD4+ T cell count in natural hosts, these infections are effectively non-pathogenic. The differences between natural and human hosts must, thus, be due to the molecular specificity of viral perturbation of the host system: that is the gain (or loss) of protein-protein interactions during adaptation to different host species or because these host systems differ themselves.

More general work on the use of the host system by pathogens [8] has found patterns in the types of interac- 
tions and infection strategies employed by multiple pathogens. Specifically, pathogens appear to preferentially interact with "key" human proteins that already participate in multiple interactions and/or have central importance in intra-cellular communication. Highly connected proteins, or "hubs", have classically characterised vulnerable points in a network due to their role in a large number of interactions and due to their association with essentiality [9-11]. Similarly, "bottlenecks", that is proteins with a high betweenness centrality, a measure of the total number of shortest paths going through the protein $[12,13]$, also associate with protein essentiality [14-16]. It has been inferred that this non-uniform contact with the host system represents evolutionary pressure to optimise exploitation of the host cell [8].

In order to test the hypothesis that the specificity of HIV interactions is in some way explained by network properties, we examine their biological context by integrating known phenotypic properties. Our analysis is based on the HIV-1, Human Protein Interaction Database (HHPID), which currently comprises over 2,500 unique interactions, curated from over 3,200 papers with over half of the interactions validated by being linked to multiple publications $[2,4]$. While this data set no doubt contains false positive interactions and potential bias, it nevertheless constitutes an excellent catalogue of HIVhuman interactions as represented by published research [3].

In terms of phenotypic properties, whilst it is difficult to assess human gene essentiality directly, we can use mouse genome knockout data as a proxy for the importance of a gene in terms of a known phenotypic consequence in disrupting its product's function [17]. Similarly, gene-disease associations from The Online Mendelian Inheritance in Man (OMIM)[18] provide another cohort of genes for which deleterious mutations are associated with phenotypic consequence. Integrating these phenotypic data into our network would be expected to corroborate any relationships with topological properties, since proteins with a high connectivity and high betweenness centrality have been demonstrated as having a tendency to be essential [9-11,14-16].

Correcting for ascertainment bias, however, we find that there is no significant relationship between HIV interaction and protein essentiality, and there is a potential under-representation of disease-association amongst HIV interacting human proteins. Moreover we find that HIV's propensity to interact with highly connected and central proteins is most probably a consequence of interactions with specific cellular functions. Thus, the biological context of HIV-interacting proteins, rather than their individual properties, has been the key determinant in the infection of hosts by retroviruses.

\section{Results}

HIV tends to interact with key host proteins such as bottlenecks and hubs

The HIV-interacting human proteins are known to interact with approximately 6,000 other human proteins when integrated with the composite human protein interaction data set from NCBI, dramatically highlighting the highly connected nature of the HIV-host interactions and their neighbours [4] (Figure 1). Proteins with a high degree (connectivity) are involved in a large number of interactions and have been previously shown to be associated with essentiality [9-11]. We used the Gene Set Enrichment Analysis (GSEA) algorithm [19] to determine if the degree distribution of HIV-interacting proteins, taken from HHPID [4], is greater than for random sets of genes taken from the human proteome. We calculate the enrichment scores (ES, from GSEA) for the degree of $\mathrm{HIV}$-interacting proteins, in addition to 10,000 random samples (each of the same size, see methods) taken from the protein-coding gene population $\left(\right.$ rand $\left._{(p o p)}\right)$. The $\mathrm{ES}_{\text {(degree) }}$ for HIV-interacting proteins was 0.83 , significantly higher than the average amongst the $\operatorname{rand}_{(\text {pop })}$ sample, 0.69 (p-value of $8.90 \times 10^{-48}$ ) (Figure 2A, grey distribution). Note, a higher ES denotes a stronger tendency towards higher degrees. This result confirms a previously reported propensity for HIV to interact with highly connected proteins [8].

However, there will potentially be substantial bias in these results due to the nature of literature curation [20]. In investigations such as this, we are reliant on accurate data to annotate our interactions. It is feasible that these genes, and their annotations, are influenced by ascertainment bias in the literature. Specifically, highly studied genes and proteins, particularly those associated with medically important molecules, can bias results. To compensate for this, we devised a novel method to evaluate ascertainment bias based on a particular gene's publication count in PubMed. If a gene has a high publication count, it is inferred that it is highly studied, and there is therefore a greater chance of observing an interaction. Randomly sampling from a population without correcting for publication counts does not offer fair comparison between the control and (biased) query samples (Figure 3). That is, HIV-interacting proteins are more likely to be highly studied and hence have had greater secondary analysis leading to greater annotation. The control set should be equally studied to offer a fair comparison. Accordingly, we calculated the ES for the degree of HIVinteracting proteins amongst 10,000 randomised samples taken to match the publication count distribution of the HIV sample $\left(\operatorname{rand}_{(l i t)}\right)$. Figure 2A (purple distribution) shows these distributions. The $\mathrm{ES}_{(\text {degree) }}$ of the HIV-interacting proteins is 0.83 and the average amongst the 


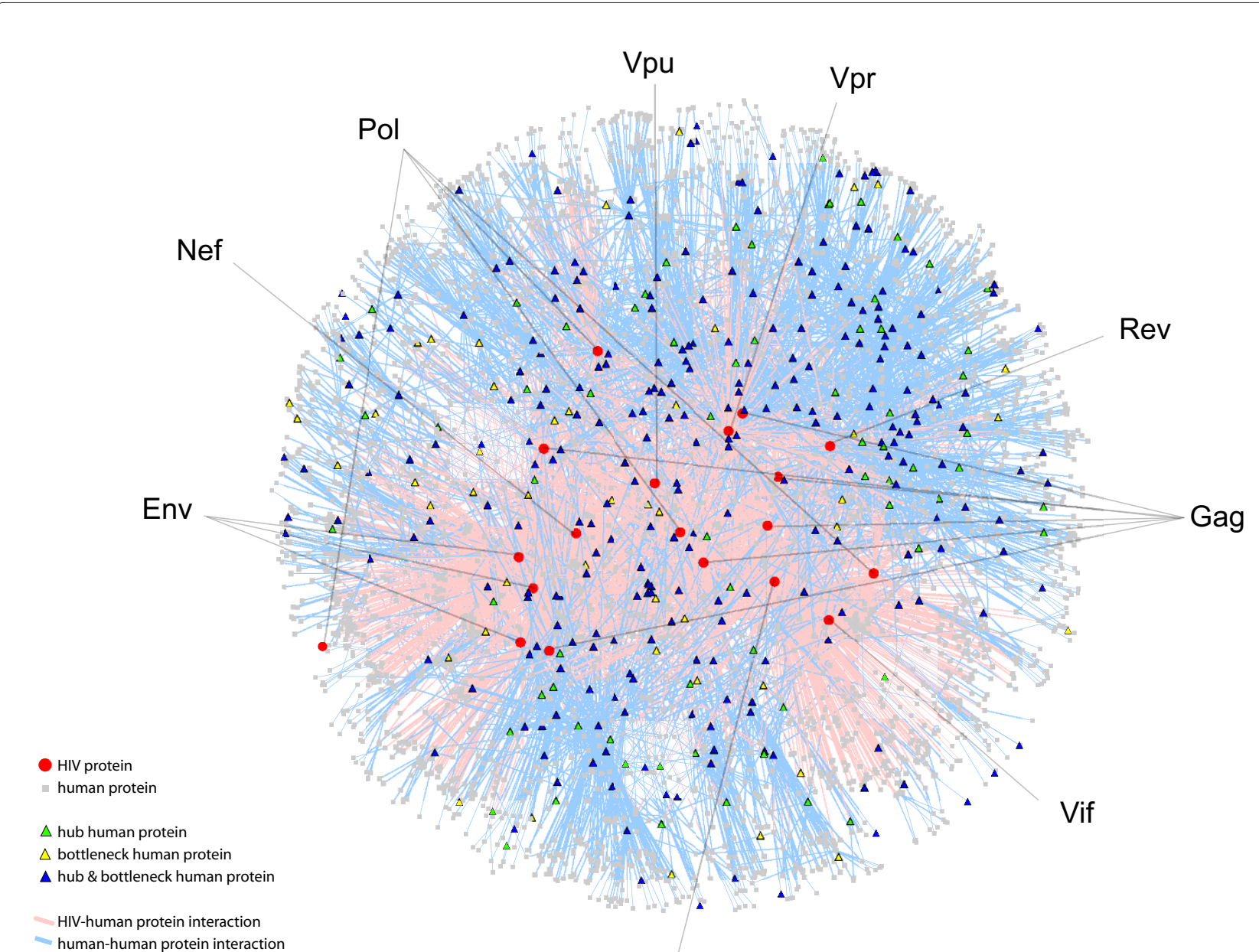

Tat

Figure 1 Subset of the HIV-host interaction network. Red circles and grey squares correspond to HIV-1 $(n=19)$ and human proteins, respectively $(n=3,118)$. Human proteins that are hubs (degree $\geq 23$ ) or bottlenecks (betweenness $\geq 2.43 \times 10^{-04}$ ) are shown as green triangles $(n=69)$ and yellow triangles $(n=45)$, respectively. Human proteins that are hubs and bottlenecks are shown as blue triangles $(n=261)$. Pink edges correspond to interactions between HIV-1 and human proteins $(n=2588)$ whilst blue edges correspond to human-human interactions $(n=3800)$, where one of the proteins interacts with HIV-1. HIV-1 proteins are labelled.

$\operatorname{rand}_{(p o p)}$ sample is 0.69 (p-value of $8.90 \times 10^{-48}$ ) whilst that of rand $_{(l i t)}$ is 0.80 (p-value $6.63 \times 10^{-15}$ ). Thus, even after correcting for ascertainment bias, we can confirm that HIV tends to interact with proteins that have a high degree.

Numerous studies have suggested that betweenness centrality also has some significance for the properties of proteins [14-16]. Does HIV preferentially interact with proteins that have a high betweenness? We calculated the ES of the betweenness centrality (in the same way as for degree), amongst the sample data sets. The $\mathrm{ES}_{\text {(betweenness) }}$ of HIV-interacting proteins is 0.90 and the average ES amongst the $\operatorname{rand}_{(p o p)}$ sample is 0.84 (p-value of $1.98 \times 10^{-}$ ${ }^{21}$ ), whilst that of rand $_{(l i t)}$ is 0.88 (p-value of $4.36 \times 10^{-8}$ ). Again, despite a significant difference between $\operatorname{rand}_{(p o p)}$ and rand $_{(i t)}$, HIV-interacting proteins can be shown to have a higher betweenness centrality than expected (Figure $2 \mathrm{~B})$.

To highlight the consequence of the betweenness centrality/degree overlap, a partial human-human protein interaction network visualisation was created using HIVhost interactions from HHPID (pink edges) and then incorporating any additional human-human interactions the human partner has (blue edges) from NCBI (see methods). This was annotated with nodes that are hubs (high degree), bottlenecks (high betweenness centrality) or both hubs and bottlenecks (Figure 1). Furthermore, HIV-interacting over-representation was demonstrated in the full network $(\mathrm{n}=21,504)$ amongst hubs but not bottlenecks $(n=92)$ and conversely bottlenecks but not 


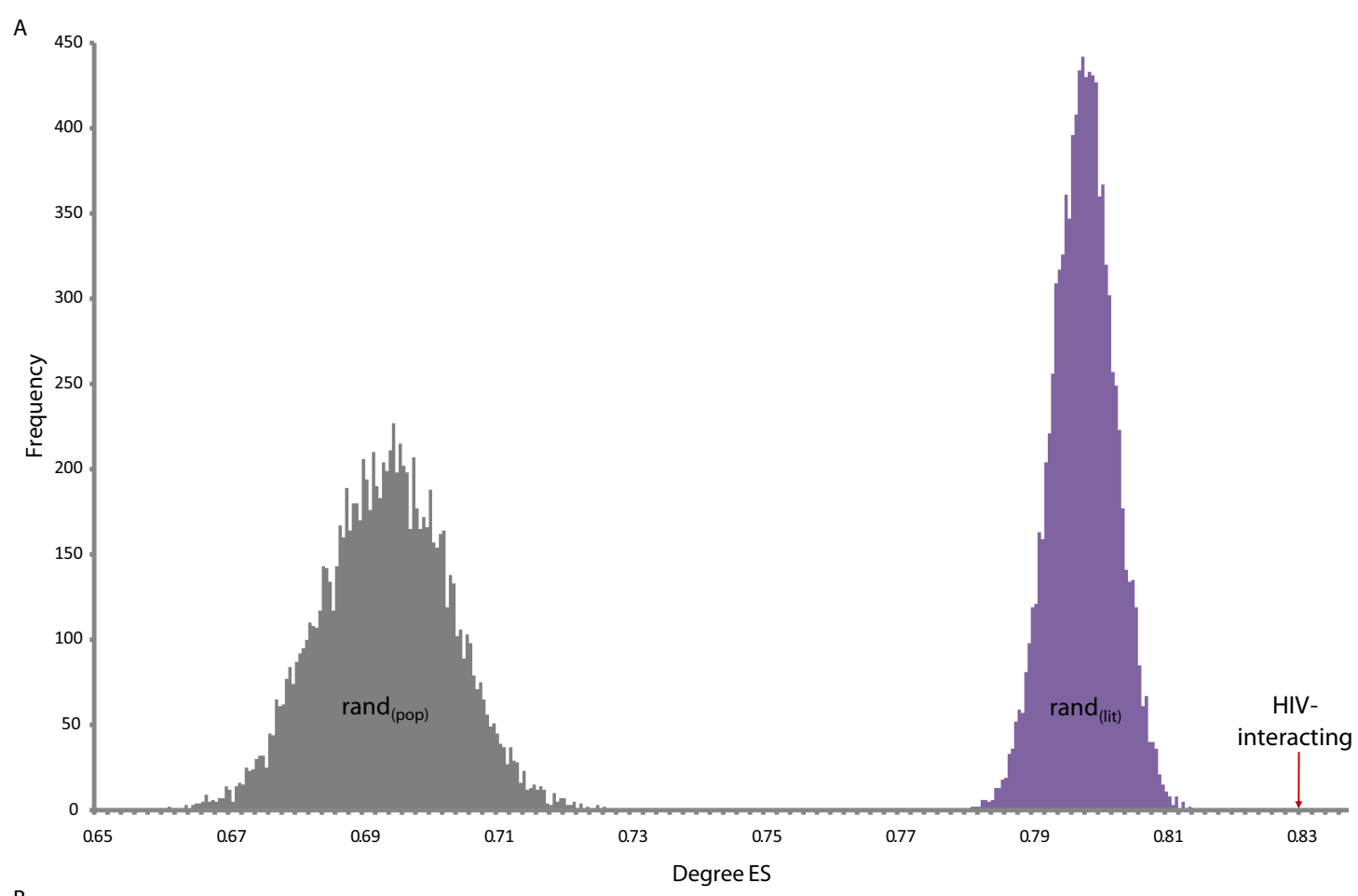

B

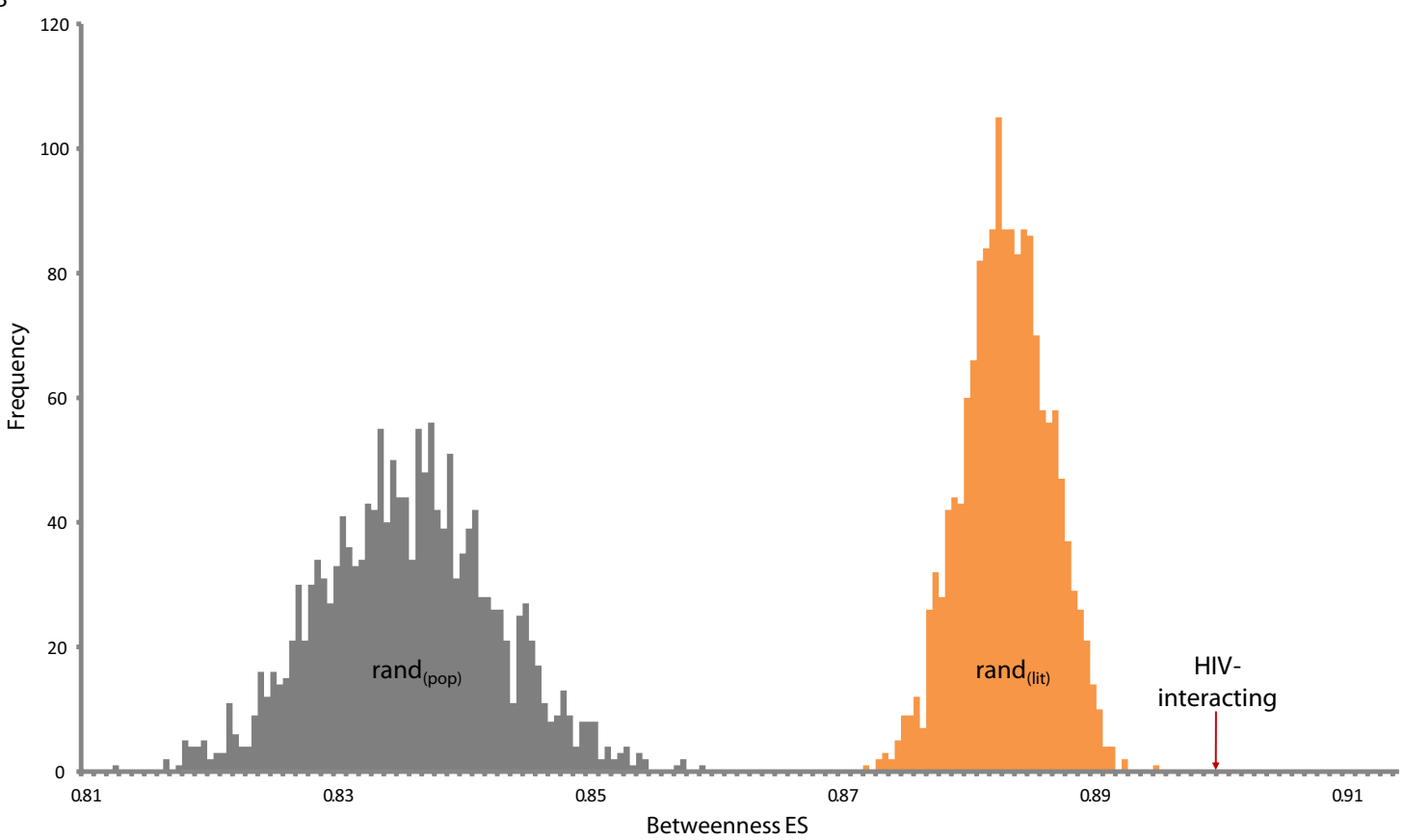

Figure 2 A) Degree enrichment amongst HIV and randomised data sets. Distributions of enrichment scores (ES), from gene set enrichment anal-

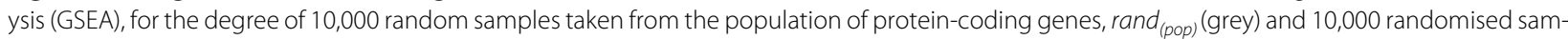

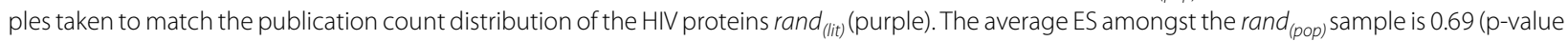
of $\left.8.90 \times 10^{-48}\right)$ whilst that of rand $d_{(i t)}$ is $0.80\left(p-v a l u e\right.$ of $\left.6.63 \times 10^{-15}\right)$. B) Betweenness enrichment amongst HIV and randomised data sets. Distri-

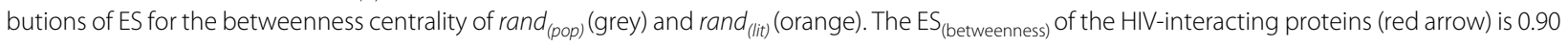

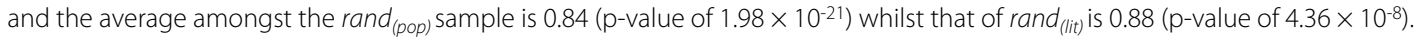




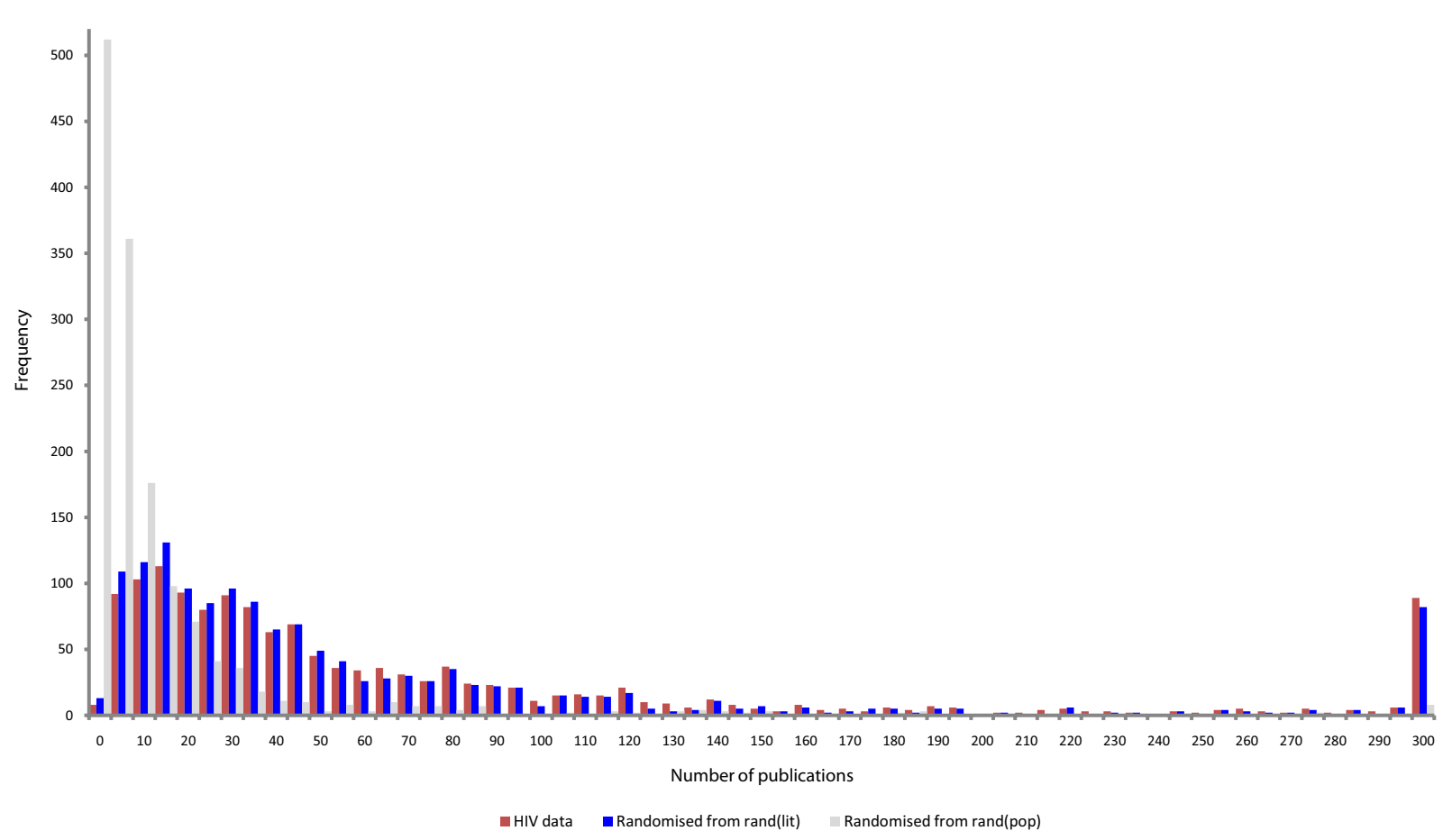

Figure 3 Rejection sampling versus random sampling. Average publication distributions for 10,000 random samples taken from the population ${\text { of protein-coding genes, } \text { rand }_{(\text {pop })} \text { (grey) and 10,000 randomised samples taken to match the publication count distribution of the HIV sample, rand }}_{(i t)}$

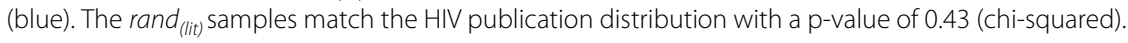

hubs $(\mathrm{n}=85)$ and was found to be $51.09 \%$ (p-value of 1.34 $\left.\times 10^{-28}\right)$ and $32.94 \%\left(\mathrm{p}\right.$-value of $\left.6.72 \times 10^{-12}\right)$ respectively.

These results raise some questions: why has HIV evolved to preferentially interact with key host proteins? Is HIV preferentially interacting with functionally "essential" proteins, as has been suggested for pathogens generally $[8]$ ?

\section{HIV-interacting genes have no relationship with essentiality}

To test this premise for the human interaction network, we explored the relationship between protein essentiality, degree and betweenness centrality, using mouse genome knockout data as a suitable proxy for essentiality [17]. We assume that a human gene can be considered essential if a knockout of its mouse ortholog confers lethality [21]. We find that there is a positive correlation between protein connectivity and essentiality (Figure 4A, p-value of $2.52 \times$ $\left.10^{-6}, \mathrm{r}^{2}=0.92\right)$ in the human protein interaction network. Similarly, a positive correlation exists between protein betweenness centrality and essentiality (Figure 4B, pvalue of $\left.5.86 \times 10^{-5}, \mathrm{r}^{2}=0.90\right)$. These relationships are unaffected by a $28 \%(n=603)$ overlap with disease-associated genes as their exclusion from the data did not alter the displayed trend (Figure 4A, p-value of $9.87 \times 10^{-6}, \mathrm{r}^{2}=$ 0.89 and $3 \mathrm{~B}, \mathrm{p}$-value of $\left.6.14 \times 10^{-5}, \mathrm{r}^{2}=0.88\right)$. This sug- gests that the greater the protein degree or betweenness centrality, the greater the likelihood of essentiality, as previously characterised [9-11,14-16].

We have confirmed that proteins with a high degree are more likely to be HIV-interacting and more likely to be essential (Figure 2 and 4). We might therefore expect to see an over-representation of essentiality amongst HIVinteracting proteins. Integrating homolog mouse genome knockout data [17] with the HIV interactions reveals that HIV interacts with 376 (26.28\%) essential proteins (Figure $5 \mathrm{~A})$. Furthermore, the mean number of essential proteins seen in rand $_{(\text {pop })}$ is only 143 (9.99\%) (Figure 5B). There is therefore an apparent overrepresentation of essentiality amongst the HIV-interacting genes (p-value of $3.86 \times 10^{-}$ 95). Investigating the PubMed statistics for essential and non-essential genes reveals that essential genes have on average a far greater publication count (mean 64 to 14 , respectively; Figure 4C). Thus, inferences about the relationships between essentiality and degree are potentially distorted. Correcting for ascertainment bias using the method previously described, the mean number of essential genes in rand $_{(\text {lit) }}$ is 399 (27.88\%), similar to the number amongst HIV-1 interacting proteins. Whilst being under-represented, the result is not significant (p-value of 0.0574). This illustrates how failure to compensate for 

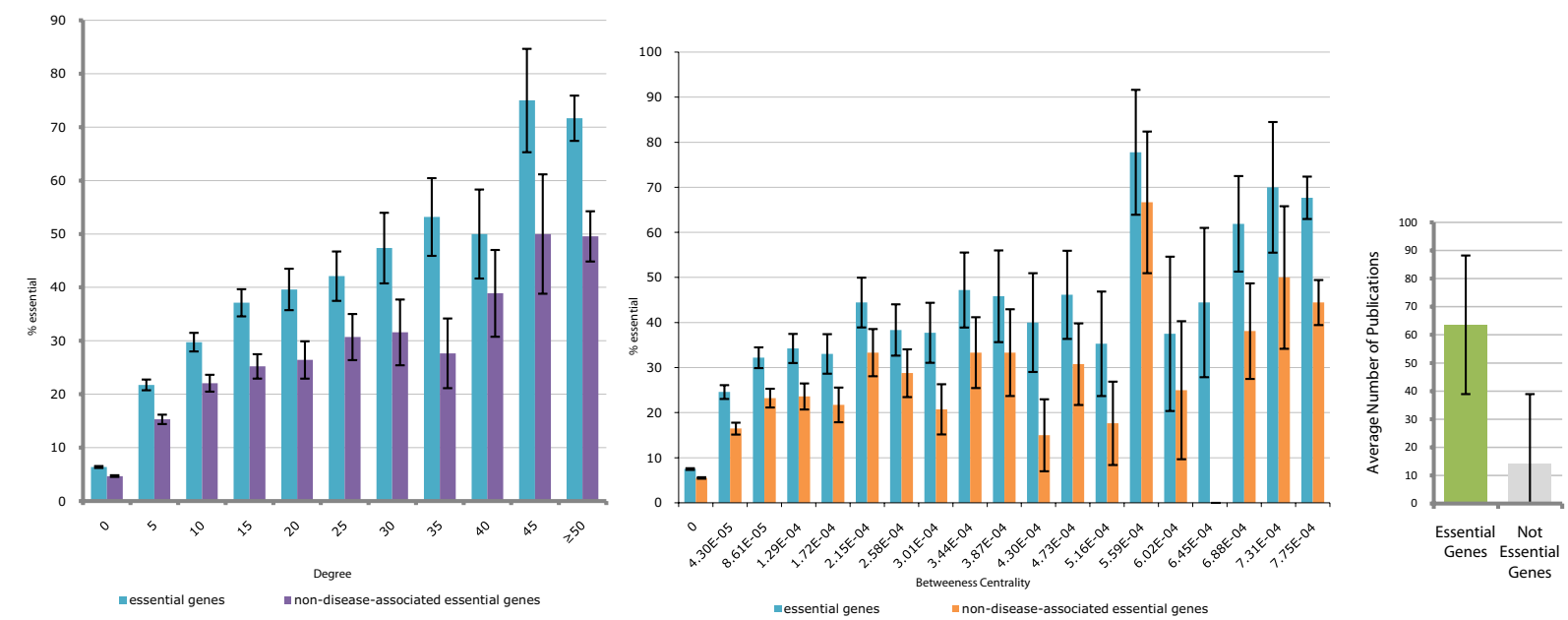

Figure 4 Essentiality and topological properties of the human network. (A) Relationship between protein degree and essentiality. Protein degree and the percentage of essential genes (blue) demonstrate a positive linear relationship ( $p$-value of $2.52 \times 10^{-6}, r^{2}=0.92$ ). Excluding overlapping disease-associated genes from the essential set (purple) does not alter the relationship ( $p$-value of $9.87 \times 10^{-6} r^{2}=0.89$ ). (B) Relationship between protein betweenness centrality and essentiality. As for panel A, but for betweenness centrality. The percentage of essential genes (blue) demonstrate a positive linear relationship with betweenness ( $p$-value of $5.86 \times 10^{-5}, r^{2}=0.90$ ). Similarly excluding overlapping disease-associated genes from the essential set (orange) does not alter the relationship, ( $p$-value of $6.14 \times 10^{-5}, r^{2}=0.88$ ). (C) Relationship between publication count and essentiality. Essential genes (green) have an average greater publication count than non-essential genes (grey): 64 to 14 publications, respectively.
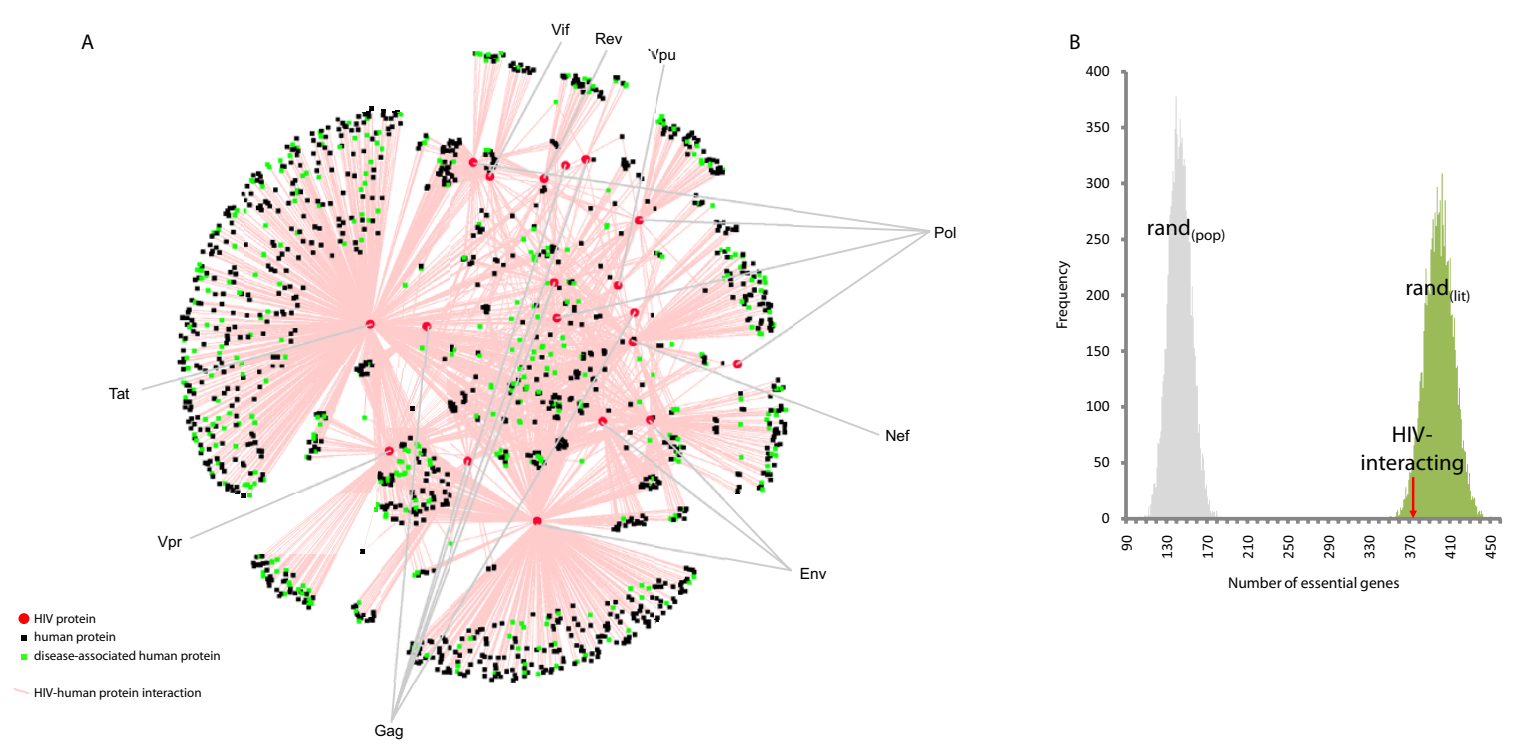

Figure 5 Essentiality and HIV-interacting proteins. (A) Visualisation of essential proteins amongst HIV-interacting proteins. Green squares correspond to human proteins identified as essential from mouse knockout data. Black squares and red circles correspond to human and HIV proteins, respectively. Pink edges correspond to interactions between HIV-1 and human proteins, as shown in Figure 4. HIV proteins are labelled accordingly. (B) Number of essential proteins amongst HIV and randomised data sets. Without correcting for bias, rand (pop) contained an average $143(9.99 \%)$ essential proteins, compared to $376(26.28 \%)$ essential proteins in the HIV set ( $p$-value of $\left.3.86 \times 10^{-95}\right)$. When the bias is corrected for, rand (iit) contains an average 399 (27.88\%) essential genes, whilst being under-represented, the result is not significant ( $p$-value of 0.0574 ) and is hence similar to the HIV-1 interacting sample. 
ascertainment bias may affect the overall result. Unexpectedly, our results therefore do not support any significant relationship between HIV-1 interaction and essentiality: HIV appears to be no more or less likely to interact with essential genes than other genes, despite their high connectivity and high betweenness centrality.

\section{HIV-interacting genes tend not to be disease-associated}

Disease associated genes have previously been shown to display a limited propensity towards encoding hub proteins [22]. To test this, we explored the relationship between disease-association and connectivity (Figure $6 \mathrm{~A}$ ), in addition to betweenness centrality (Figure 6B). We find that, whilst there is a slight positive correlation between degree and disease-association (Figure 6A, pvalue of $\left.0.02, r^{2}=0.47\right)$, this disappears when the $33 \%(n=$ 603) overlap with essentiality is removed (Figure 6A, pvalue of $\left.0.29, r^{2}=0.13\right)$. Disease-association and betweenness centrality (Figure 6B, p-value of $0.01, \mathrm{r}^{2}=0.33$ ) are also less correlated when the overlap is removed (Figure $6 \mathrm{~B}$, p-value of $0.45, \mathrm{r}^{2}=0.04$ ).

Without correcting for bias, it appears that gene disease association obtained from OMIM [18] is significantly over-represented amongst HIV-interacting proteins when compared to non-HIV-interacting proteins (Figure 7). Of the HIV-1 interacting human genes, 244 (17.05\%) are associated with disease, whilst $120(8.39 \%)$ of the randomised samples $\left(\operatorname{rand}_{(p o p)}\right)$ on average are disease-associated $\left(\mathrm{p}\right.$-value of $\left.8.58 \times 10^{-32}\right)$. Investigating the publication count in PubMed for disease-associated and non-disease-associated genes reveals that disease-associated genes generally have larger numbers of publications (mean 67 versus 16; Figure 6C), again indicating potential bias. Correcting for this, the literature-count matched random set $\left(\right.$ rand $\left._{(l i t)}\right)$ has a mean of $336(23.48 \%)$ diseaseassociated genes ( $\mathrm{p}$-value of $3.48 \times 10^{-12}$ ). Contrary to the initial result, this suggests that there may in fact be fewer interactions with disease-associated genes than expected (Figure 7B).

\section{HIV-interacting proteins are over-represented for fundamental biological processes}

To place our findings in a stronger biological context, we next investigated the relationship between HIV-host interactions and protein function. A functional understanding of the host-pathogen interaction network can be gained by integrating annotations from GO $[4,23]$. To investigate HIV's use of the host system in more detail, we identified biological processes over-represented for HIV interactions (see also Pinney et al. [3]). These categories represent diverse functions exploited by multiple interactions, involving multiple HIV genes, demonstrating that HIV proteins co-ordinate to target specific parts of the human cellular system.

To investigate HIV's propensity to interact with key proteins, we determined the degree and betweenness centrality for proteins involved in the over-represented biological process $\mathrm{GO}$ terms, including immune and

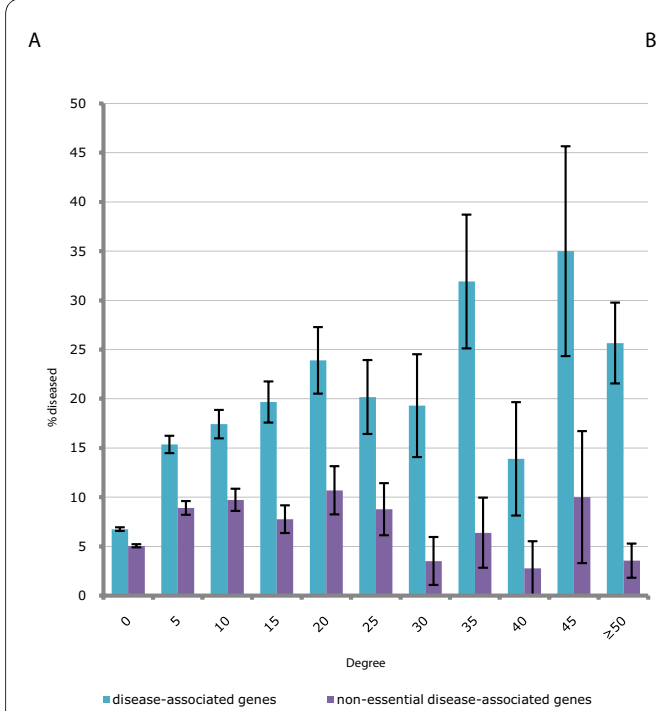

Figure 6 Disease association and topological properties of the human network. (A) Relationship between protein degree and disease-association. Protein degree and the percentage of disease-associated genes (blue) demonstrate a slight positive linear relationship ( $\mathrm{p}$-value of 0.02 ). Excluding overlapping essential genes from the disease-associated set (purple) removes the relationship ( $p$-value of 0.29). (B) Relationship between protein betweenness centrality and disease-association. As for panel A, but for betweenness centrality. The percentage of disease-associated genes (blue) demonstrate a positive linear relationship with betweenness, ( $p$-value of 0.01). Similarly excluding overlapping essential genes from the disease-associated set (orange) removes the relationships, (p-value of 0.45). (C) Relationship between publication count and disease-association. Disease-associated genes (green) have an average greater publication count than non-disease-associated genes (grey), 67 to 16 publications, respectively. 


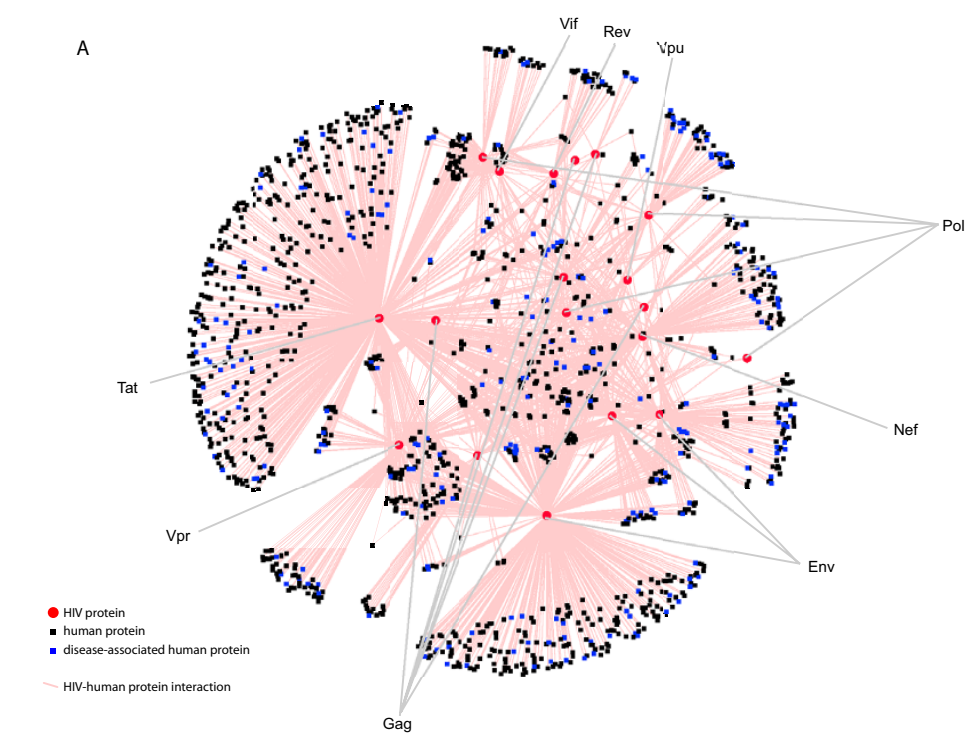

B

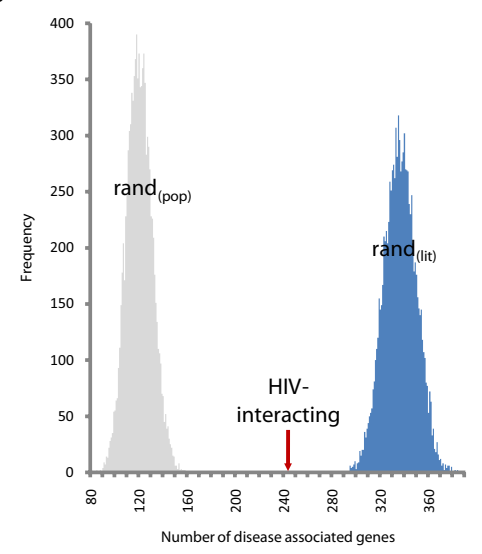

Figure 7 Disease association and HIV-interacting proteins. (A) Visualisation of disease-associated genes amongst HIV-interacting proteins. Blue squares correspond to human proteins identified in OMIM as being disease-associated. Black squares and red circles correspond to human and HIV proteins, respectively. Pink edges correspond to interactions between HIV-1 and human proteins, as shown in Figure 4. HIV proteins are labelled accordingly. (B) Number of disease associated genes amongst HIV and randomised data sets. Without correcting for bias, rand (pop) contained an average 120 (8.39\%) disease-associated genes, compared to 244 (17.05\%) disease-associated genes in the HIV set ( $p$-value of $8.58 \times 10^{-32}$ ). When the bias is

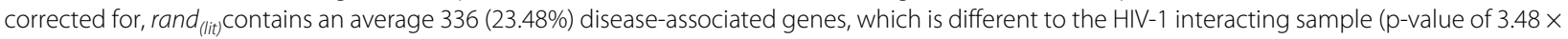
$10^{-12}$.

apoptotic processes (Figure 8). We find proteins with these terms are generally more highly connected and central. The mean degree and betweenness centrality amongst the human protein interaction network was found to be 2.63 and $2.33 \times 10^{-5}$, respectively, whilst those of the functionally over-represented proteins are 7.27 and $7.40 \times 10^{-5}$, respectively. This finding that human proteins within the functional classes that HIV interacts with most often are themselves more likely to be hubs and bottlenecks accounts for the observed tendency of HIV-interacting proteins to be highly connected and central and is independent of the genes' essentiality or disease association.

\section{HIV's use of the host system}

The HHPID data include the specific type of interaction between HIV and host proteins, for instance up- or down-regulation[3,4]. Combining this information with GO permits an analysis of how the various HIV genes perturb multiple host processes. We classified the 68 interaction types from HHPID into three polar categories: positive (denoted by +) for 'activated by', 'activates', 'enhanced by', 'enhances', 'stabilizes', 'stimulated by', 'stimulates', 'upregulated by' and 'upregulates'; negative (denoted by -) for 'cleavage induced by', 'cleaved by', 'cleaves', 'competes with', 'degraded by', 'degrades', 'disrupts', 'downregulated by', 'downregulates', 'inactivates', 'induces cleavage of', 'inhibited by' and 'inhibits'; with the remaining 25 interaction types as neutral (denoted by / ) $[2,4]$. For most of the over-represented biological processes GO terms, we find the majority of interactions are mainly 'positive' in nature. The only exception, not surprisingly, is the immune response for which the majority of interactions are more 'negative' in nature (Figure 9). This demonstrates that HIV perturbs multiple cellular processes in multiple ways, that is, HIV appears to be both up- and down-regulating a wide range of proteins and functions.

\section{Discussion}

Our results confirm that HIV preferentially interacts with hubs and bottlenecks - key host proteins that are apparently important to the cell (Figures 2 and 4). As proteins with a high connectivity and high betweenness centrality have previously been shown to demonstrate a tendency towards being essential [9-11,14-16] (and see Figures 4A and $4 \mathrm{~B}$ ), we investigated whether selection for interactions with essential proteins could account for these network topological observations. This was done by integrating phenotypic data - assessed with protein essentiality inferred from mouse knockout data - into our analysis. After correcting for ascertainment bias, however, we found no significant relationship between HIV-1 

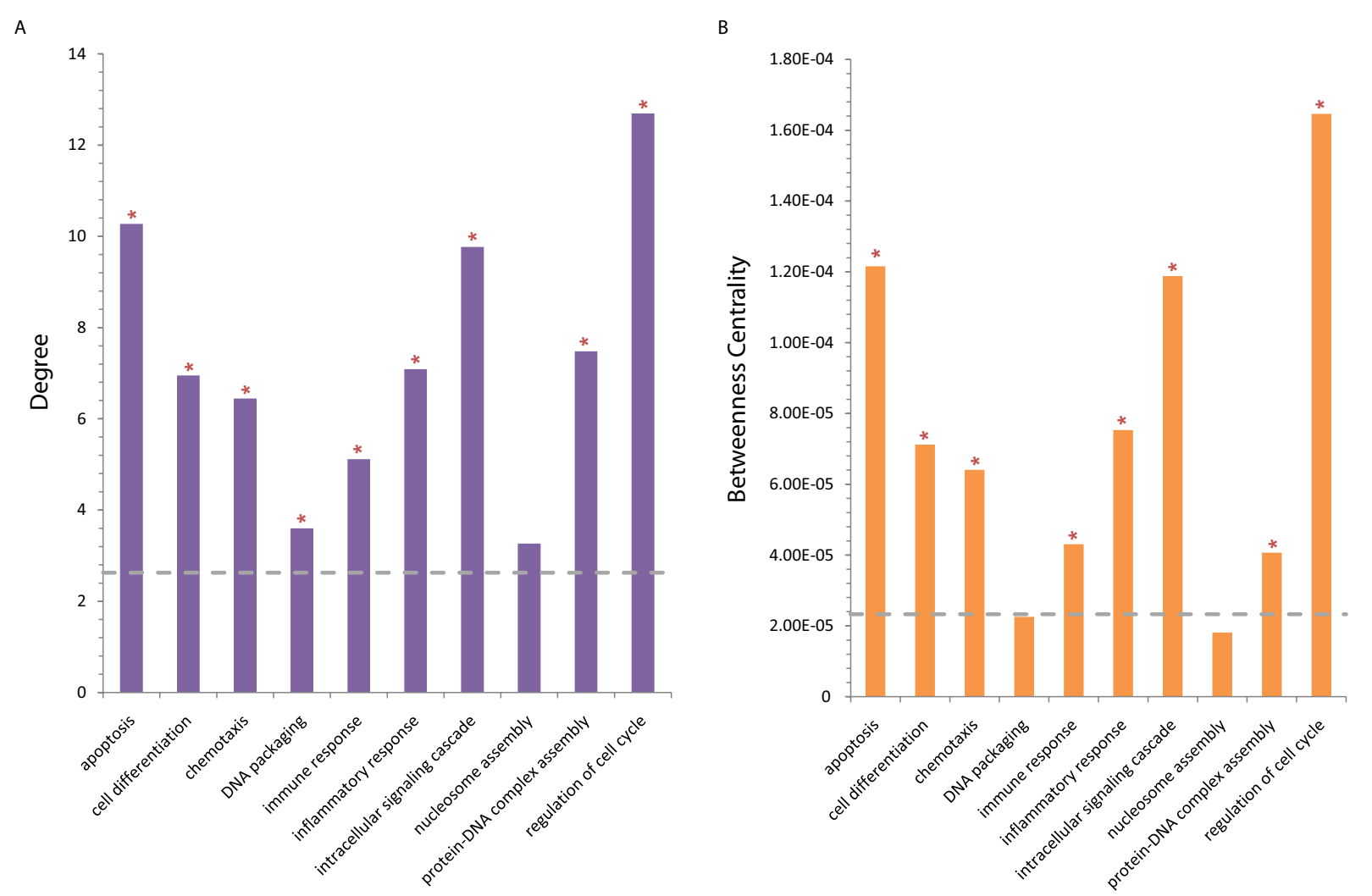

Figure 8 Protein degree (A) and betweenness centrality (B) for proteins involved in key cellular processes. The degree and betweenness centrality for proteins involved in the key over-represented biological processes GO terms from [4]. Grey dashed lines correspond to the average degree (2.63) and betweenness centrality $\left(2.33 \times 10^{-5}\right)$ amongst the human-human protein interaction network. The functionally over-represented proteins have a mean degree and betweenness of 7.27 and $7.40 \times 10^{-5}$, respectively. P-values (from Wilcoxon rank-sum test) $<0.05$ are indicated by an asterisk $\left(^{*}\right)$ above the data-points, suggesting the degree/betweenness distribution for each $\mathrm{GO}$ term is significantly different than that for all $\mathrm{GO}$ terms.

interaction and protein essentiality (Figure 5B). That is, HIV-1 proteins appear to be no more or less likely to interact with essential proteins than expected by random chance. This lack of over-representation of interactions with essential proteins (despite a significant tendency to interact with key host proteins) could be the result of ancestral selection pressure on retroviruses to minimise interactions with phenotypically essential proteins. Specifically, this would be consistent with selection acting on HIV's retroviral ancestors (due to longstanding co-evolution of retroviruses with host species) to minimise the pathogenic outcome of infection and maximise transmission potential, presumably in a trade-off between virulence and transmissibility [24,25].

Using an alternate measure of phenotype associated with perturbation: disease association, we investigated these observations further. Disease genes have previously been shown to display no propensity towards encoding either lowly or highly connected proteins [22] and we find that this is also true of the human protein interaction network when the overlap with essential genes is removed
(Figure 6A and 6B). Accordingly, we would expect to observe no relationship between disease-association and HIV interaction amongst human proteins. Initially we find an over-representation of disease-association amongst HIV-interacting human proteins (Figure 7B). However, after compensating for ascertainment bias in the literature, we find the opposite: there appears to be an under-representation of disease-association amongst HIV-interacting proteins (Figure 7B). As there is no apparent relationship between connectivity and diseaseassociation (Figure 6A), the under-representation of disease-association amongst HIV-interacting proteins is not related to network topology. Rather, we hypothesise that this under-representation of disease-association could again represent a selection pressure on retroviral proteins to avoid interacting with proteins associated with adverse phenotypes.

Given these results, how can we explain HIV's tendency to interact with high-degree and high-betweenness host proteins? Dyer and co-workers [8] have suggested that viral and bacterial proteins tend to interact with key pro- 


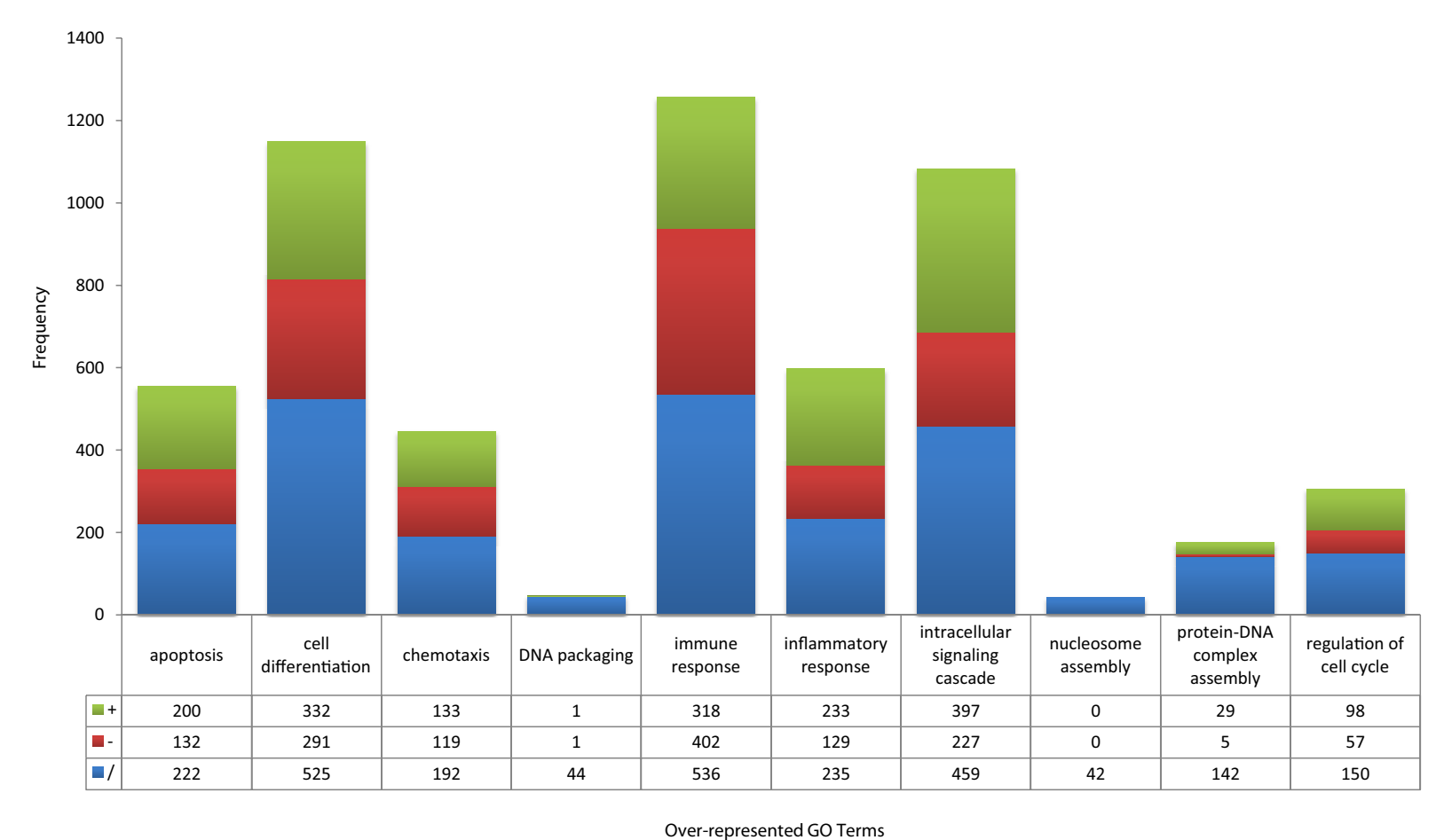

Figure 9 Directionality of HIV-host interactions by functional category. The frequency of interaction types classified as positive (green), negative (red) or neutral (blue) according to functional categories over-represented for HIV interactions; see Results for further details.

teins, as they may control critical human cellular processes, through their high connectivity and betweenness centrality. We find that the two concepts are interrelated: certain human proteins are central because they represent essential cellular functions, e.g., immune response. HIV interacts with these proteins to achieve its biology, and their high connectivity is simply secondary to this. Indeed, proteins involved in the over-represented biological process GO terms tend to be highly connected and central (Figure 8). Thus, HIV's propensity to interact with highly connected and central proteins is mainly a consequence of its interactions with particular cellular functions, rather than being related to global network properties in any straightforward way.

The specificity of the HIV-1 host interaction from HHPID, in the context of these underlying host protein functions, permits a detailed analysis of HIV's perturbation of the host system. Indeed, focussing on biological functions (from GO), our analysis demonstrates the directionality and complexity of both pro-pathogen (the majority promoting HIV's replication cycle) and pro-host (the host response to infection) interactions with specific cellular functions [3]. Collectively this highlights the subtle but complex manipulation of the host cell.

Throughout our analyses, we have corrected for the potential effect of ascertainment bias [20]. However, as it is very difficult to provide an accurate estimate for the degree of bias in the HHPID data, we have deliberately chosen a very conservative methodology for bias correction. Therefore, whilst we can be confident that degree and betweenness are both higher than expected after correction, it is possible that we are over-correcting in the case of the essentiality and disease-association data. Our results should therefore be interpreted as indicating no evidence for over-representation of these properties amongst HIV-interacting proteins; further research into bias correction methods for genome-scale data will be needed in order to provide more definitive conclusions.

\section{Conclusion}

In order to fully understand HIV's hijack of the host system it will be necessary to study in detail the functional modules that are being exploited. This is exemplified by the complexity of HIV-host interactions, with the same functions being targeted multiple times (Figure 9). It will also be important to study the directionality of interactions, i.e., those that are pro-pathogen interactions as opposed to pro-host interactions[3], or even bystander interactions, incidental interactions of little consequence to either virus or host. Our finding that that there are patterns in terms of the types of interactions HIV makes can be explained by the cellular functions that HIV requires in order to replicate. The apparent tendency for HIV to 'avoid' phenotypically important molecules, underlines - 
despite HIV's recent acquisition by humans - the longstanding relationship that retroviruses have with their hosts. As more data become available, it will be informative to study this co-evolution of pathogens with their (often changing) host species. Understanding the precise molecular specificity of both the adaptation and persistence of pathogens with their hosts will yield novel insights into virulence and, potentially, new intervention strategies.

\section{Methods}

\section{Main Data sets}

Human protein interactions were derived from multiple sources: BioGRID http://www.thebiogrid.org, BIND http://www.bind.ca and HPRD http://www.hprd.org and filtered from the NCBI "interactions" file ftp:// ftp.ncbi.nlm.nih.gov/gene/GeneRIF. Interaction data contained in these data sets are derived from multiple sources. HIV-host interactions and properties were derived from The HIV-1, Human Protein Interaction Database (available at http://www.ncbi.nlm.nih.gov/RefSeq/HIVInteractions). The data set currently comprises 1,435 human genes encoding 1,448 proteins that interact with 19 HIV-1 proteins making 2,589 unique interactions, curated from over 3,200 papers published between 1984 and 2007[2,4]. This paper also made extensive used of the "gene_info" and "gene2refseq" files provided by the Entrez Gene database ftp://ftp.ncbi.nlm.nih.gov/gene filtered to human genes $(\mathrm{n}=36,455)$ and limited to those known to be protein-coding $(\mathrm{n}=21,504)$. All data sets were current as of July 2009.

\section{Protein Essentiality}

To predict the essentiality of a human gene, we used the phenotype information of the corresponding mouse ortholog. A human gene was defined as essential if a knockout of its mouse ortholog confers lethality. We obtained the human-mouse orthology and mouse phenotype data from Mouse Genome Informatics http:// www.informatics.jax.org/[17]. We considered the annotations of postnatal, prenatal and perinatal lethality as lethal phenotypes, and the rest of the phenotypes as nonlethal ones. Overall, 27,697 annotations were filtered to leave 2,145 genes with an inferred essentiality.

\section{Disease Association}

The Online Mendelian Inheritance in Man (OMIM) Morbid Map http://www.ncbi.nlm.nih.gov/sites/ entrez? $\mathrm{db}=$ omim contains the most complete curated disorder-gene associations [18]. The data was filtered for the "(3)" tag http://www.ncbi.nlm.nih.gov/Omim/omimfaq.html\#gene_map_symbols, for which there is strong evidence that at least one mutation in the particular gene is causative to the disorder, to identify 3,328 unique dis- eases across 3,049 genes. We used the gene_info file (to convert OMIM gene symbols to NCBI GeneIDs to facilitate integration. This data was used as a proxy for mild phenotypic effect.

\section{Gene Ontology}

GO terms [23] were collected for each human gene from the NCBI "gene2go" file ftp://ftp.ncbi.nlm.nih.gov/gene/ DATA. Term ancestors were then determined for each term from "gene_ontology_edit.obo" http://www.geneontology.org to ensure complete coverage. Select GO terms were taken from $[3,23]$, retested for over-representation amongst HIV-interacting human proteins using Fisher's Exact Test in R[26] and separated into the three ontologies: biological process, cellular component and molecular function.

\section{Network Visualisations}

Networks were visualised as graph-based layouts using Cytoscape [27].

\section{Degree, Hubs, Betweenness and Bottlenecks}

The degree of a vertex in a network is the number of connections it has, in the case of a PPI network, this represents the number of other proteins the vertex interacts with. The degree of a single vertex is therefore equal to the number of adjacent edges.

A protein with a high degree is considered a hub and these have frequently been identified as the most vulnerable points in biological networks [9-11,14-16]. Yu et al. [16] classify a protein as a hub if it falls within the top $20 \%$ of proteins when sorted according to their degree. A cutoff of $20 \%$ in our data categorises a hub as any protein with a degree $\geq 3$, we therefore chose a stricter cut-off of $2 \%$ so a hub is only classified as such with a degree $\geq 23$.

Betweenness is a centrality measure of a vertex within a graph that summarises its relative importance both locally and globally [14-16]. Vertices that occur on many shortest paths between other vertices have higher betweenness than those that do not and are considered bottlenecks. Bottlenecks are generally a more accurate indicator of essentiality than degree or hub propensity[16], despite the two being correlated. For a graph $G=$ $(V, E)$, the betweenness centrality $C_{B}(v)$ for vertex $v$ is: $C_{B}(v)=\sum_{\substack{s \neq v \neq t \in V \\ s \neq t}} \frac{\sigma_{s t}(v)}{\sigma_{s t}}$, where $\sigma_{s t}$ is the number of geodesic (shortest) paths from $S$ to $t$, and $\sigma_{s t}(v)$ is the number of geodesic paths from $S$ to $t$, that pass through a vertex $v$. We use Brandes' algorithm[12] to calculate the betweenness centrality of all vertices in $G$, normalised by dividing through the number of pairs of vertices not including $v$ : $(V-1)(V-2)$. As for hubs, we define a bottleneck as the top 
$2 \%$ of ranked proteins, so a bottleneck is classified as such with a normalised betweenness centrality $\geq 2.43 \times 10^{-4}$.

The Wilcoxon rank-sum test, implemented in $\mathrm{R}$ [26], was used to compare the distributions of degree/ betweenness across the entire genome against individual over-represented biological processes GO terms (see above). This enables us to determine whether the distributions for each GO term are significantly different to that found in the genome.

\section{Ascertainment bias}

For every protein coding gene contained in Entrez Gene $(\mathrm{n}=21,504)$, we obtained the number of unique publications using "Entrez Programming Utilities" http:// eutils.ncbi.nlm.nih.gov/entrez/query/static/

eutils help.html. In total, 409,964 publications were recorded; with an average 19 articles per gene $(2,217$ genes were not matched to a publication).

Rejection sampling was used to generate sets of random genes that matched the publication frequency distribution of the HIV-interacting human set $f(x)(\mathrm{n}=1,431)$, from the overall protein-coding gene population with publication frequency distribution $g(x)(\mathrm{n}=21,504)$.

The John von Neumann Monte Carlo algorithm [28] was used, such that instead of sampling directly from the distribution $f(x)$, we use an envelope distribution $M g(x)$, where $M$ is the maximal $f(x)<M g(x)$, and selected such that $f(x)<M g(x)$ for all observed publication counts $x$ :

A) A gene (with publication count $x$ ) is selected at random from the overall population with publication frequency distribution $g(x)$. A random number $U$ from $U(0,1)$ is also selected.

B) If $u<\frac{f(x)}{M g(x)}, x$ is accepted as a realisation of $f(x)$

and the gene is kept, otherwise sample step (A) is repeated.

The procedure is repeated until a set of genes of the required size is obtained. The samples match the distribution with a p-value of 0.43 (chi-squared, Figure 3). Using this procedure we constructed 10,000 sets of 1,431 randomised genes, rand $_{(i t)}$, matching the publication frequency distribution of the HIV-interacting human genes. For comparison, 10,000 fully randomised samples, $\operatorname{rand}_{(p o p)}$, were also generated by standard random sampling from the set of all genes. When comparing observed properties to these random samples, a z-score calculation was used to standardise the raw score $s$ of each property tested, $z=\frac{s-\mu}{\sigma}$ and this was converted to a P-value using $R$ [26]. This enables us to determine whether any results in the HIV-interacting set are due to ascertainment bias.

\section{Gene set enrichment analysis}

Following the example of Dyer et al. [8], we adapted the gene set enrichment analysis (GSEA) method of Subra- manian et al. [19] to test for significant differences between HIV-interacting and random sets of genes (both $\operatorname{rand}_{(l i t)}$ and $\left.\operatorname{rand}_{(p o p)}\right)$. For a graph $G=(V, E)$ let $L$ be the list $V$ ranked by either degree or by betweenness centrality. Let $S$ be a subset of vertices within $L$, for example, the vertices that are HIV-interacting, rand $_{(l i t)}$ or $\left(\right.$ rand $_{(p o p)}$. Let $l_{i}$ be the value (of degree or centrality) at index $i$ of $L$, such that $1 \leq i \leq|L|$. If $i$ is a member of $S$, the protein whose rank is $i$, thus, belongs to $S$. First, calculate $m=\sum_{i \in S} l_{i}$, the sum of all the values of $S$. Next, for each index $i$ of $L$, we compute two values, $P_{h i t}(S, i)=\sum_{j \in S, j \leq i} \frac{l_{j}}{m}$, the weighted fraction of proteins in $S$ with an index $\leq i$ and $P_{\text {miss }}(S, i)=\sum_{j \notin S, j \leq i} \frac{1}{|L|-|S|}$, the fraction of proteins not in $S$ with an index $\leq i$. The enrichment score is therefore the largest positive value of $e s(S, L)=P_{\text {hit }}(S, i)-P_{\text {miss }}(S, i)$. A large positive value of $e s(S, L)$ indicates that the proteins in $S$ have high degree or high betweenness centrality. To compute p-values for the observed es $(S, L)$, Dyer and coworkers [8] selected $|S|$ random proteins from $L$ $1,000,000$ times and estimated the p-value based on this distribution. However, we predict $S$ to be biased, so similarly biased random samples $|S|$ must be taken from $L$. We therefore used rejection sampling to generate 10,000 samples of $\left|S_{H I V}\right|$ with the distribution of $S_{H I V}$ in preference to the naïve random selection. A p-value was calculated from the $\mathrm{z}$-score using $\mathrm{R}[26]$.

\section{Authors' contributions}

All authors participated in the design of the study. JED carried out all analysis and drafted the manuscript. JWP helped with the statistical analysis. DLR conceived of the study. All authors edited and approved the final manuscript.

\section{Acknowledgements}

JED is supported by a Wellcome Trust studentship and JWP by a Royal Society University Research Fellowship. Thanks also to the Apple Research \& Technology Support scheme for support.

\section{Author Details}

'Faculty of Life Sciences, University of Manchester, Oxford Road, Manchester, M13 9PT, UK and 2Division of Molecular Biosciences, Imperial College London, South Kensington Campus, London SW7 2AZ, UK

Received: 1 October 2009 Accepted: 7 June 2010

Published: 7 June 2010

\section{References}

1. UNAIDS/WHO: AIDS epidemic update. 2007 [http://data.unaids.org/ pub/EPISlides/2007/2007 epiupdate en.pdf].

2. Fu W, Sanders-Beer BE, Katz KS, Maglott DR, Pruitt KD, Ptak RG: Human immunodeficiency virus type 1 , human protein interaction database at NCBI. Nucleic Acids Res 2009:D417-422.

3. Pinney JW, Dickerson JE, Fu W, Sanders-Beer BE, Ptak RG, Robertson DL: HIV-host interactions: a map of viral perturbation of the host system. AIDS 2009, 23(5):549-554.

4. Ptak RG, Fu W, Sanders-Beer BE, Dickerson JE, Pinney JW, Robertson DL, Rozanov MN, Katz KS, Maglott DR, Pruitt KD, et al.: Cataloguing the HIV type 1 human protein interaction network. AIDS Res Hum Retroviruses 2008, 24(12):1497-1502. 
5. Chan DC, Kim PS: HIV entry and its inhibition. Cell 1998, 93(5):681-684.

6. Weiss RA: How does HIV cause AIDS? Science 1993, 260(5112):1273-1279

7. Heeney JL, Dalgleish AG, Weiss RA: Origins of HIV and the evolution of resistance to AIDS. Science 2006, 313(5786):462-466.

8. Dyer MD, Murali TM, Sobral BW: The landscape of human proteins interacting with viruses and other pathogens. PLoS pathogens 2008, 4(2):e32.

9. Jeong H, Mason SP, Barabasi AL, Oltvai ZN: Lethality and centrality in protein networks. Nature 2001, 411(6833):41-42.

10. Yu H, Greenbaum D, Xin Lu H, Zhu X, Gerstein M: Genomic analysis of essentiality within protein networks. Trends Genet 2004, 20(6):227-231

11. He $X$, Zhang J: Why do hubs tend to be essential in protein networks? PLoS Genet 2006, 2(6):e88.

12. Brandes U: A Faster Algorithm for Betweenness Centrality. Journal of Mathematical Sociology 2001, 25(2):163-177.

13. Freeman LC: Centrality in Social Networks I: Conceptual Clarification. Social Networks 1979, 1:215-239.

14. Hahn MW, Kern AD: Comparative genomics of centrality and essentiality in three eukaryotic protein-interaction networks. Mol Biol Evol 2005, 22(4):803-806.

15. Joy MP, Brock A, Ingber DE, Huang S: High-betweenness proteins in the yeast protein interaction network. J Biomed Biotechnol 2005, 2005(2):96-103.

16. Yu H, Kim PM, Sprecher E, Trifonov V, Gerstein M: The importance of bottlenecks in protein networks: correlation with gene essentiality and expression dynamics. PLoS Comput Biol 2007, 3(4):e59.

17. Eppig JT, Blake JA, Bult CJ, Kadin JA, Richardson JE: The mouse genome database (MGD): new features facilitating a model system. Nucleic acids research 2007:D630-637.

18. Hamosh A, Scott AF, Amberger JS, Bocchini CA, McKusick VA: Online Mendelian Inheritance in Man (OMIM), a knowledgebase of human genes and genetic disorders. Nucleic Acids Res 2005:D514-517.

19. Subramanian A, Tamayo P, Mootha VK, Mukherjee S, Ebert BL, Gillette MA, Paulovich A, Pomeroy SL, Golub TR, Lander ES, et al:: Gene set enrichment analysis: a knowledge-based approach for interpreting genome-wide expression profiles. Proceedings of the National Academy of Sciences of the United States of America 2005, 102(43):15545-15550.

20. Pfeiffer T, Hoffmann R: Temporal patterns of genes in scientific publications. Proc Natl Acad Sci USA 2007, 104(29):12052-12056.

21. Liao BY, Zhang J: Null mutations in human and mouse orthologs frequently result in different phenotypes. Proceedings of the National Academy of Sciences of the United States of America 2008, 105(19):6987-6992.

22. Goh Kl, Cusick ME, Valle D, Childs B, Vidal M, Barabasi AL: The human disease network. Proceedings of the National Academy of Sciences of the United States of America 2007, 104(21):8685-8690.

23. Harris MA, Clark J, Ireland A, Lomax J, Ashburner M, Foulger R, Eilbeck K, Lewis S, Marshall B, Mungall C, et al:: The Gene Ontology (GO) database and informatics resource. Nucleic Acids Res 2004:D258-261

24. Bull JJ: Perspective - Virulence. Evolution 1994, 48:1423-1437.

25. Holmes EC: On the origin and evolution of the human immunodeficiency virus (HIV). Biol Rev Camb Philos Soc 2001, 76(2):239-254

26. R-Development-Core-Team: R: A language and environment for statistical computing. R Foundation for Statistical Computing. Vienna, Austria; 2008

27. Shannon P, Markiel A, Ozier O, Baliga NS, Wang JT, Ramage D, Amin N, Schwikowski B, Ideker T: Cytoscape: a software environment for integrated models of biomolecular interaction networks. Genome Res 2003, 13(11):2498-2504.

28. Neumann J: Various techniques used in connection with random digits. Monte Carlo methods. Nat Bureau Standards 1951, 12:36-38.

\section{doi: 10.1186/1752-0509-4-80}

Cite this article as: Dickerson et al., The biological context of HIV-1 host interactions reveals subtle insights into a system hijack BMC Systems Biology $2010,4: 80$

\section{Submit your next manuscript to BioMed Central} and take full advantage of:

- Convenient online submission

- Thorough peer review

- No space constraints or color figure charges

- Immediate publication on acceptance

- Inclusion in PubMed, CAS, Scopus and Google Scholar

- Research which is freely available for redistribution 